\title{
The Reaction of the Planets Upon the Sun-I"
}

\author{
Influence of the Earth and a Study of Sun Spots
}

\section{By P. Puiseux,' Member of the Institute, Astronomer at the Paris Observatory}

THE popular preconception that the earth, with the sun rotating about it, was the center of the universe, was overcome only through the persistent efforts of astronomers and physicists. We will not here revie these memorable discussions, but will note merely the result. Everyone capable of connected and geometr cal reasoning will become convineed that the position of the earth, face to face with the sun, is that of a humble satellite, and that our globe, forced to escort our daytime star in its mysterious course through space, receives from this star its law of annual movement and at the same time its indispensable ration of heat and light

Going from one extreme to another, the sun was be lieved to be independent of the relatively minute planet which it carries along with itself. It seemed that fictitious observer, placed at its center or on its surface would have no occasion to suspect the existence of other celestial bodies. Further protected against any perceptible action from the stars by their immense distance the sun must lavish its splendor, with no pay in return and follow unperturbed its undeviated path through and foll

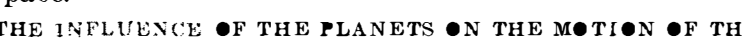

This conclusion was in some respects too radical. An account of this matter could be rendered only when the penetrating genius of Newton showed that the curved trajectory of a projectile, the revolution of the moon about the earth, and the revolution of the earth around the sun were three manifestations of the same law. Thi law holds everywhere. Further, it is not a special privilege of the center of any system. The bond exists, real thougb slight, between any two particles whatever. The sun, as well as the humblest planet, because of this bond, must undergo periodic variations in its speed as well as in its shape.

Ha ve we to-day at our disposal sufficient delicate mean of observations to detect these changes? In Newton's time such means were probably lacking. The caprices of our atmosphere furnished a ready explanation of the apparent fluctuations in solar radiation. The spots had been observed on the sun's disk, sometimes few, some times many, but no law had been assigned to them. Further, the traditional fixity of the constellations led to the with reference to the stars.

But the problem plainly stated aroused new attempts at its unraveling. Bradley, a fellow countryman and a disciple of Newton, showed that much greater precision could be obtained in the measures of the angular distances of the stars than had before been gained. Less than a century later, W. Herschel could affirm that the constellations do alter their form, and the best determination of these changes may be explained by attributing to the solar system a regular rectilinear motion. The ambition of astronomers, increasing with success, tries to-day to show that this movement is not rigorously uniform, and even though shielded from the action of the stars, and even though shielded from the action of the stars,
pays tribute to the universal attraction in periodic oscillations.

It is pretty safe to predict what will be the most marked of these oscillations. It is not the center of the sun itself which possesses the uniform rectilinear motion, but the center of gravity of the system formed by the sun and all the planets. The oscillation would be small if only the earth need be considered. There is, however, a the earth need be considered. There is, however, a
giant planet, Jupiter, whose mass exceeds that of all of the other planets taken together and is nearly $1 / 1,000$ that of the sun. Describing its orbit at the rate of 12 kilometers per second, Jupiter forces the sun to rotate about an imaginary center with a velocity a thousand times less. This is apparently a very small amount, but not at all negligible with respect to the velocity of translation of the solar system, which is 20 kilometers per second. Consequently, the speed of the solar system per second. Consequently, the speed of the solar system accelerated, sometimes slowed, by one part in one thouaccelerated, sometimes slowed,
sand in an interval of 6 years.

Very few of the stars are near enough to us for the parallactic displacement relative to the more distant stars and due to this motion of the sun to be appreciable in 6 years. Consequently, to measure $1 / 1,000$ part of this displacement is beyond the resources of precise astronomy. We may be pretty sure, though, that some day we will thus obtain, at the same time with a measure of the mass of Jupiter, a sat sfartory n'w confirmation of

Hecture delivered at the Comservatioire des Arts et Mêtiers,
lehruary 23rd. 1913. le bruary 23rd, 1913

Translated from Revue Scientifique, Paris, May 3rd, 1913, in the Andual Report of the Smithsonian Institution. the principle of the universal attraction of gravitation. Meanwhile help comes in another way. What the micrometer for a long time will probably be unable to
give, the spectroscope is always furnishing. Although the variation of 30 meters per second, which we wish to detect in the motion of the sun, requires years to change sensibly the apparent position of a star, it takes only a moment to alter the quality of its light. Whatever the moment to alter the quality of its light. Whatever the
distance, the light waves will come to us sometimes distance, the light waves will come to us sometimes
more frequently, sometimes less; their path through a prism will consequently be found altered and the fine metallic lines of the spectrum recorded by a photograph will be displaced relatively to those of a stationary source, such as an electric spark used for comparison.

The earliest happy applications of this principle wer due to Huggins and to Vogel. It was used to separate numerous double stars composed of pairs of suns so close numerous double stars composed of pairs of suns so close
to each other and so distant from us that each pair apto each other and so distant from us that each pair ap-
peared as a single star. But the brightness of each was peared as a single star. But the brightness of each was
sufficient to record a spectrum and the relative velocities were sufficiently variable so that two spectrum lines of the same chenical origin separated periodically. Subsequently another class, yet greater in number, was found a periodic oscillation. In this case we may suppose that one of the two stars, while not bright enough to register its spectrum, is yet heavy enough to sway its associate. The period is usually several weeks or days. The displacements of the lines correspond to velocities of the same order as those of the planets, from 10 to 100 kilometers per second. Because of the extreme accuracy and care in the use of spectroscopes, certain astronomers The time will come when pairs like the sun and Jupiter can be detected, however distant they may be, provided only that the principal star is bright enough to record only that the principal star is bright enough to record
its spectrum. Campbell, who is the leader in this. class its spectrum. Campbell, who is the leader in this. class
of researcb, estimates that on the average one star in three will be found spectroscopically double. It is very probable that even more stars are double since we can see no reason why a planet like Jupiter should be excepfound thus variable even that all stellar spectra will be movement of the earth. We may then gather photographic evidence of the existence of planets about the graphic evidence of the existence of planets about the
stars as well as the periodic oscillation of our sun due to stars as well as the periodic oscillation of our sun due to
Jupiter. The earth, of course, will produce a similar effect only less in amplitude and period. But who would dare to put a limit to the skill of our opticians or the patience of our astronomers in a path so definitely marked out?

To find that as THE CAUSE $\bullet$ F THE SOLAR CYCLE. To find that we disturb the sun is of course something tion if we can find that we cause changes in the aspect tion if we can find that we cause changes in the aspect
of its surface, disturbances visible by direct and not of its surface, disturbances visible by direct
indirect evidence in the field of the microscope.

We will now consider a deforming action dependent also on Newton's law but of a differential nature and consequently proportional to the inverse cube instead of the inverse square of the distance. This difference helps to compensate for the inferiority of the mass of the earth with reference to the greater planets and

chance for an honorable rank in this contest.

We have under our eye an encouraging phenomenon. The attraction at the surface of the earth due to the sun is but a small fraction compared to the weight of a body
here. and the yet feebler attraction due to the moon can not lighten a body by 100/1,000 part of its weight. $\mathrm{Y}$ et we see the moon exerting this power and indeed with three times more strength than is felt from the sun, in deforming our globe. This action can be detected upon the atmosphere, the oceans, and even the solid crust of the earth. The seas, however, are what rende it most evident to our eyes. Under favorable cond iions, for instance, in the Bay of Mont St. Michel, on the French coast, we see the sea following faithfully
the passage of the moon across the meridian. The sea's level changes at the flood some 20 meters in a few hour displacing the shoreline several kilometers. The wor thus developed, if we could only put it to use economically, would be enough to render useless all our oil wells and all the engines in the world.

We may find that no planet is as favorably situated to trouble the sun as the moon is the earth. But perhaps we should not he so exarting. We sce upon the su no such liquid seas wirich might be made to extend or comtract their Ammains. 'The weight there to be con that, we see chances that the sun may react as actively, or even more actively, than the earth, under the action of a distant body. We are indeed led by several converging patbs of reasoning to think that the surface layers tenuous mobile matter, little subject to the action of weight and all ready, consequently, to obey the least force.

A first piece of evidence along this line is the development of spots, rents which seem to appear in the luminous veil of the solar surface, reaching in a few days an extent of 10,20 , or 30 thousand kilometers and disappearing with equal rapidity. In the spectrum of these spots there is an increase in the number and intensity of the absorption bands, leading us to think that are spouted out in torrents, carried along by currents of lighter hydrogen.

More impressive yet is the appearance of protuberances-clouds which develop and remain at heights where they could not be sustained by the dense and refringent atmosphere. Much less bright than the disk, they have a special spectrum and during total eclipses are the principal source of light. We can now photoingenious method devised in 1868 by Janssen and by Lockyer and since singularly perfected. On many occasions we have been assured by incontestable evidence that protuberances can mount in a few hours in the form of vertical jets, narrow at the base to prodigious heights $-50,000$ to 100,000 kilometers or even more. Generally, however, before attaining such heights the protuberances expand into sheaves or stratified layers. At times they seem to be the seat of violent explosions, are scattered. and disappear very quickly. The spectroscope shows us that calcium vapor, despite its atomic weight 40 times heavier than that of hydrogen, rises very high in the protuberances. The displacements of the spectrum lines also furnish confirmation of the enormous velocities (100 kilometers or more per second) which the deformations of the contours suggest.

Total eclipses, during which protuberances first attracted attention, are even now the only occasions when we can see another interesting phase of solar activitythe solar corona. Sometimes it appears as a halo somewhat equally distributed around the disk, at other times as gigantic streamers stretching out distances several times the diameier of the sun. The forms of these rays indicate that the matter of which they are composed shows no haste in falling back into the sun. This matter is evidently very sparse and has very little absorptive action on light, for, despite its irregular distribution, it causes no difference in the appearance of the various parts of the disk. Its mobility must be very the various parts of the disk. Its mobility must be very eclipses its structure completely changes, as our photographs assure us.

Spots, protuberances, and corona are subject to a grea variation which takes place regularly about nine times in a century. After a period when the sun's disk appears entirely immaculate, spots re-appear in both then, always increasing from 20 degrees to 30 degrees, regions, becoming at the maximum 20 times more numerous on the average than in a minimum year Then, as the decline commences, the numerical predominance which the Northern Hemisphere at first seemed to sbow passes to the Southern Hemisphere. The spots firs disappear in the high latitudes and then diminish al over the sun.

The protuberances pass through a similar sycle, except that during the period while their number increases their mean latitude tends to increase in each hemisphere. Toward the epoch of spot maximum, and only then, it is not rare to see great protuberances even near the poles, where spots never appear

The corona during the same period always undergoes a definite evolution. Toward the epoch of sun-spot minimum the polar rays are fine and vertical like the bristles of a brush. The jets in the middle and mean latitudes are much longer and bent toward the Equator. At the maximum period there is little difference with the A thitude. During: the transition years the poles and the laitude. During: the transition years the poles and the
Equator are almost clear and the rays are developed only in the middle latitudes, giving the whole a reclangular appearance.

The more we reflect upon these farts the less are wo led to regard the sun as a monarch, inaceessible, and shut up in a tower of ivory. It, like the earth, must have and tides connected with its own rotation. To sift 
out at least the more active of these external influences is a legitimate task, even though it is not an easy one. First, do we find one or several bodies which could be held responsible for a cycle of 11 years? The stars seem held responsible for a cycle of 11 years? The stars seem to be beyond consideration, since in that period there is
no appreciable change in their linear or angular distances. We could, as did John Herschel. blame one or several swarms of meteors, imagined for the purpose. Describing very eccentric orbits, they might graze the surface of the sun, causing the spots. Suitably choosing their revolution periods, inclinations, eccentricities, and the distribution of the matter in their orbits, we could confess that the permanence of swarms of meteors confess that the permanence of swarms of meteors
put every 11 years to such a violent test does not seem put every 11 years to such a violent test does not seem
probable. There is no doubt that meteors fall into the sun in great numbers. But we have no direct proof that this happens periodically and so as to produce visible effects. Such proof we feel that we must demand for this very supple and convenient hypothesis. As these swarms have not been detected, we must leave them and direct our investigations to the planets.

The most important of these planets brings a coincidence at first sight very seductive. Nearly every 11 years Jupiter, in a determinate sense, crosses the plane of the solar equator; also in every 11 years the numerical predominance of the spots passes from the northern to the southern bemisphere of the sun. The same interval separates the return of Jupiter to its least distance from the sun and the return of the sun-spot. numbers to their extreme value.

We must not hurry, though, to sign our victory. It is not an approximate concordance but a precise one which we should demand. The periods in years are 11.86 for the revolucion of Jupiter and 11.13 for the sunspot cycle. For the second period which is less wel
defined, the incertitude is in the hundredths. For more than a century we have careful records of spot number which reappear regularly. Now, in the course of century the difference of 8 months between the periods brings them from complete coincidence to an absolute discordance. What now remains of our hoped-for proof if the nearest approach of the planet must sometimes if the nearest approach of the planet must sometimes
condition an increase of spots, sometimes their discondition an

We may suppose that Jupiter's action, though pre ponderant, is modified by a somewhat slower disturbing force which increases the interval between successive maxima. But the statistics of the number and exten force, assigns to it such a long period that we have no force, assigns to it such a long period that we have no
clue as to its origin. A priori the most probable disclue as to its origin. A priori the most probable dis-
turbing body would seem to be Saturn. It must act turbing body would seem to be Saturn. It must act
in the same sense as Jupiter, although to less extent The spot maxima or minima should be particularly pronounced when the two planets are in conjunction with the sun - that is, every 20 years. Here again the evidence is negative.

We get an even less favorable answer from the rest of the planets. Either their revolution periods are too short to render an account of an 11-year fluctuation or their distances too great for their action to be sensible compared with that of Jupiter.

The planets as a isturbing element in the sola CYCLE

No planet then, or combination of planets seems to be the principal cause of the solar cycle. We may, however, suppose that this or that planet may for a brie time trouble the cycle by rend
of spots irregular in longitude.

The sun rotates with reference to the fixed stars once The sun rotates with reference to the fixed stars once
in 25 days. The planets revolve about it in the same direction, but more slowly. Therefore, to an observer on the sun, the successive passages of a planet over his meridian occur in periods somewhat longer than 25 days, rending to approach this (sidereal revolution) as the planet's distance increases. This is called the synodical rotation. That corresponding to the transit of the earth is 2735 days.

Considering now the extreme mobility of the solar surface, we will see whether each planet does not produce a tidal wave which passes over the sun's surface with the corresponding synodical rotation period and capable of producing visible disturbances.

According to the elementary law of Newton, the relative importance of the tidal waves for the various planets is given by what we may call the deforming factor, the product of the mass by the inverse cube of the distance. If we make the value of this factor unity for the earth,

the mean values for the planets are as follows:
Mercury ............

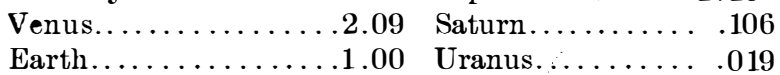

Earth

1.00 Uranus.

019

We see that the most active cause for a tidal wave lies in Jupiter, followed closely by Venus. Mercury and the earth come next, the remaining planets being much lesis active.

Although the earth comes only in the fourth rank,

we will consider it first because we are better situated for examining its effects. At each instant we can consider the sun as divided into two equal hemispheres, ne visible, the other not. The limiting meridians tum uniformly over the surface of the sun in 27.35 days, the ynodical period.

Let us first suppose that the earth has no physical influence on the development of the spots. The racio between the total sun-spot areas in the two hemisphere may happen to have any value whatever; but the mean value taken over a long period of time embracing man synodical rotations, say for a whole solar-spot cycle. synodical rotations, say for a who
should differ very little from unity.

We can not at any given moment count or measure the spots on the invisible hemisphere. But we can count the spots which appear on the eastern border and com pare these with those which disappear in the corresponding time limit at the western border. The ratio of the two numbers would have a tendency to surpass unit if it is at a time of decrease in spots and to be less than unity if in the increasing phase. But taken over a whole cycle, the mean value should differ very little from unity.
Now let us suppose that the earth does have a physical influence, for instance, to fix our attention, that the presence of the earth above the horizon of some poin on the sun favors the development of a spot at that point. As this development is certainly not instantaneous, any more than is its disappearance, more spots one. Consequently, more spots will disappear over the western border than appear at the eastern. The in western inequality will be observed, provided we observe verse inequality will be observed, provided we observe
over a sufficiently long period, if the presence of the earth causes the disappearance of spots.

Inslead of comparing the eastern with the western disk, the right with the left, and the result would be equally decisive. Practically, if the action of the earth on the solar surface is real, the action will necessarily take a certain time to become manifest. Considerable take a certain time to become manifest. Considerable
masses must be moved, masses doubtless subject to interior friction. It is so relative to terrestrial tides which at any point of the earth suffer a variable retardation, but. always very marked with reference to the passage of the moon over the meridian. If the earth has no influence, the two halves--the right and leftwould, if considered over a sufficient time, show the same number and same area of spots. If the earth has a real influence there
tematic inequality.

RESEARCHES $\bullet$ F MRS. MAUNDER, 1907.

Mrs. Maunder undertook to answer this question, utilizing the photographs due to a co-operation of English observatories for the interval 1889 to 1901 , extending from one spot minimum to the next. At the beginning and the end the sun seemed absolutely free from spots. at the beginning and the end of the period upon the visible hemisphere could not vitiate the conclusions derived from all the observations.

The tables obtained at Greenwich comprised-

(1) The positions and areas of the groups for each day. (2) The history, day by day, of each important group; the areas are expressed in millionths of the visible hemisphere and are corrected for the effcet of perspective;
the mean duration of a group. is about 6 days; 2,870 groups were studied.

Mrs. Maunder divided the visible hemisphere at each instant into 14 vertical zones, each 13.2 degrees wide and numbered in the inverse order of their appearance. For each zone and the entire period the sum representing the area of the spots was made. These results were compared for zones symmetrical to the central meridian. There was thus made manifest a systematic variation from two points of view:

(1) Despite the perspective correction, there was a limb to the central zone, as if the perspeciive correction had been insufficient

(2) For each pair of zones there was a constant decrease in passing from the eastern to the correspondin western zone. The same thing was noted when in similar manner the northern and souchern hemisphere were treated separately.

Various reasons make the measures on the extreme zones less trustworthy; but even if we omii them the same conclusions result. If refraction in the solar atmosphere plays a part it would unduly enrich the extreme zones. Accordingly, if a correction is made extreme zones. Accordingly, if a correction is made for it, it but increases the first anomaly. Neither
anomaly can be due to errors of observation or reduction. If we do not like this process of treatment we need not depend upon the areas of the spots, but count simply the number of groups visible in each zone, omitting those
of long life which necessarily appear in both halves. Here again, for all pairs of zones, the eastern one shows a greater number than its corresponding western one. We next ask whether there is, either in the visible in the invisible half, an habitual and systematic excess in the number of spot births over deaths. A priori, it seems as if it must be so for one or the other hemis phere during the phase of increasing spots, but that an equilibrium must be established when a complete cycle is considered.

To throw light on this point Mrs. Maunder associated on each half of the disk the two extreme zones and compared the number of groups of spots which had been seen in each of the two double zones. The predominance was clearly in the eastern pair. There are throughou a cycle more spots seen near the eastern border, and consequently for the whole visible hemisphere and whole cycle there is an excess of disappearances over appearances of spots. The opposite nust hold on the invisible bemisphere, since at the beginning and end of a cycle the sun is entirely free from spots.

Neglecting the extreme zones, where the disappearfor each zone the number of spots which were seen in it for the first time and the number seen in it for the last time. The following result was noted:

As we go from east to west, crossing the visible hemisphere, there is an almost constant diminution in the number of spot appearances over a whole spot cycle and as nearly constant and even greater augmentation in the number of disappearances.

When we compare two symmetrical regions of the disk, the number of births found in one is generally smaller than the number of disappearances in the corresponding region on the other side of the central meridian.

If we were dealing only with numbers, the departures

If we were dealing only with numbers, the departures
noted might be considered as resulting from a psycholonoted might be considered as resulting from a psycholo-
gical cause. It is probable that there is in an observer gical cause. It is probable that there is in an observer
a certain, perhaps unconscious, laziness which keeps him from recording new appearances and prolonging old spots unless absolutely necessary. It is always more agreeable to register a disappearance which simplifies work rather than an appearance which augments it.

Thus, when a new small spot appears for the first time, there is a tendency to include it among those time, there is a tendency to include it among those
already noted rather than to regard it as an advance guard or germ of a new group. If the first impression is wrong, then there results an unjustified diminution of births in the visible hemisphere.

In a similar manner, if a small group approaches a important group, either by expansion or derivaand to cease counting it as soon as the separation between it and the larger group ceases to be distinct We are thus led to credit fictitious disappearances to the visible hemisphere.

Both these considerations lead us to record more disappearances than births. But these errors in counting do not explain why the total area of spots is regularly found greater in the eastern half of the visible disk. Considering all of Mrs. Maunder's results we are led to think that the presence of the earth above the horizon of a place on the sun tends to make spots there disappear. ( $T$ o be concluded.)

\section{Selenium Cell Making}

Within a late period almost all selenium cells are made by using a flat slab of insulating material with double winding of rather tine platinum wire, upen which the selenium is spread. While many commercial cells use steatite as a base, amateurs will find this mate-

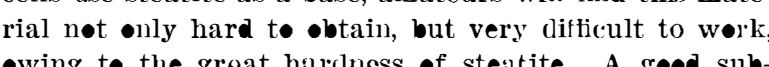
-wing to the great harr(ness of steatite. A good sub stitute is slate, in the ordinary thin slabs, which can lie easily sawed in small oblong Iieces, and then these

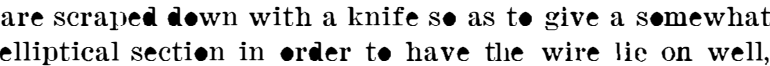
the edge being ratler fine, but not enoush sø to cause breaking. Great care should be taken to have a clean fresh surface and not to impregnate the very pørøu slate with impurities by handling with the fingers, which would spoil the insulating properties. One of the main things in a cell is to have the wire mathematically spaced. Grooves are cut along in the edges only (not -n the surface), but it is out of the question t॰ make than by machine. Of course a skilled person could easily design a cutter wheel machine for such purnose but any amateur can make a sufficient cutter by mount ing a flat wood block or cell-holder to travel along bebase is fastened a small block carrying an inserted fixed nut in which moves a piece of threaded rod; on the outer end of the rod is a large wond disk, while the inner end pushes the carriage along upon turning the screw. The cell is clamped on the block carriage. Half a turn of the screw thus drives the carriage along sa $y$ -ne millimeter each time. The edge of the cell projects -ver the carriage. Cutting could be done by a small fixed block very near the edge of the slite and use it as a guide for a flat saw blade, by which very accurate cutting can be done 